\title{
Article
}

\section{A Liouville's Formula for Systems with Reflection}

\author{
Santiago Codesido ${ }^{1,+}$ and F. Adrián F. Tojo ${ }^{2, *,+}+\mathbb{C}$
}

1 Analytical Sciences Group, Faculty of Sciences, Université de Genève, 1206 Geneva, Switzerland; santiago.codesido@unige.ch

2 Instituto de Matemáticas, Universidade de Santiago de Compostela, 15705 Santiago de Compostela, Spain

* Correspondence: fernandoadrian.fernandez@usc.es

+ These authors contributed equally to this work.

check for

updates

Citation: Codesido, S.; F. Tojo, F.A. A Liouville's Formula for Systems with Reflection. Mathematics 2021, 9, 866. https://doi.org/10.3390/ math9080866

Academic Editor: Krzysztof Ciepliński

Received: 4 January 2021

Accepted: 14 April 2021

Published: 15 April 2021

Publisher's Note: MDPI stays neutral with regard to jurisdictional claims in published maps and institutional affiliations.

Copyright: (C) 2021 by the authors. Licensee MDPI, Basel, Switzerland. This article is an open access article distributed under the terms and conditions of the Creative Commons Attribution (CC BY) license (https:// creativecommons.org/licenses/by/ $4.0 /)$.

\begin{abstract}
In this work, we derived an Abel-Jacobi-Liouville identity for the case of two-dimensional linear systems of ODEs (ordinary differential equations) with reflection. We also present a conjecture for the general case and an application to coupled harmonic oscillators.
\end{abstract}

Keywords: ODEs (ordinary differential equations); reflection; linear systems; Liouville's formula

MSC: $34 C 14$

\section{Preliminaries}

There has been recent interest in the study of differential equations with reflection. We highlight the importance of research on qualitative aspects, such as the existence and uniqueness of solution [1-3], boundedness [4] or periodicity [5]. There has also been a search for Hilbert bases related to operator eigenfunction decompositions [6,7] and explicit solutions or the associated Green's functions [8-13]. Many of these works highlight the strong relation between linear analysis and linear algebra, either in the context of ordinary differential equations [10,12], systems [13,14], difference equations [15] or partial differential equations [16]. In particular, in [13], the authors developed an explicit fundamental matrix for the system of differential equations with reflection:

$$
H u(t):=F u^{\prime}(t)+G u^{\prime}(-t)+A u(t)+B u(-t)=0, t \in \mathbb{R},
$$

where $n \in \mathbb{N}, A, B, F, G \in \mathcal{M}_{n}(\mathbb{R})$ and $u: \mathbb{R} \rightarrow \mathbb{R}^{n}$.

Theorem 1 ([13]). Assume $F-G$ and $F+G$ are invertible. Then:

$$
X(t):=\sum_{k=0}^{\infty} \frac{E^{k} t^{2 k}}{(2 k) !}-(F+G)^{-1}(A+B) \sum_{k=0}^{\infty} \frac{E^{k} t^{2 k+1}}{(2 k+1) !}
$$

where $E=(F-G)^{-1}(A-B)(F+G)^{-1}(A+B)$, is a fundamental matrix of problem (1). If we further assume $A-B$ and $A+B$ are invertible, then $E$ is invertible and we can consider a square root $\Omega$ of E. Then:

$$
X(t)=\cosh \Omega t-(F+G)^{-1}(A+B) \Omega^{-1} \sinh \Omega t .
$$

This theorem draws a parallelism between the classical theory of ODEs and that of ODEs with reflection, which puts forward the question of whether we can deepen into this comparison. In particular, we wonder whether the well-known Liouville's formula for ODEs has a counterpart in the theory of linear ODEs with reflection. We will provide a partial answer to that question in this paper, present a conjecture for the general case and analyze the particulars for systems of two dimensions. 
In what follows, we will denote by $|M|$ the determinant of a square matrix $M \in$ $\mathcal{M}_{n}(\mathbb{C})$. We recall now Cayley-Hamilton's theorem.

Theorem 2 (Cayley-Hamilton). Let $M \in \mathcal{M}_{n}(\mathbb{C})$ and consider the characteristic polynomial of $M$ defined as $p(x):=|x \operatorname{Id}-M|$. Then $p(M)=0$.

Assume $M \in \mathcal{M}_{n}(\mathbb{C})$, and the spectrum of $M$ is $\operatorname{sp}(M)=\left\{\lambda_{1}, \ldots, \lambda_{n}\right\}$. Then, the characteristic polynomial is:

$$
p(x)=\prod_{k=1}^{n}\left(x-\lambda_{k}\right)=\sum_{k=0}^{n}(-1)^{k} \mathcal{I}_{k}(M) x^{n-k},
$$

where:

$$
\mathcal{I}_{0}(M)=1, \mathcal{I}_{k}(M)=\sum_{1 \leq j_{1}<\cdots<j_{k} \leq n} \lambda_{j_{1}} \ldots \lambda_{j_{k}}, k=1, \ldots, n
$$

The $\mathcal{I}_{k}$, only depending on the eigenvalues of $M$, are invariants of $M$ under conjugation by invertible matrices. In particular, $\mathcal{I}_{1}=\operatorname{tr}(M)$ and $\mathcal{I}_{n}(M)=|M|$.

As a direct consequence of Cayley-Hamilton theorem, we have that:

$$
\sum_{k=0}^{n}(-1)^{k} \mathcal{I}_{k}(M) M^{n-k}=0,
$$

and taking the trace (which is linear) on both sides:

$$
\sum_{k=0}^{n}(-1)^{k} \mathcal{I}_{k}(M) \mathcal{I}_{1}\left(M^{n-k}\right)=0
$$

If we consider a differentiable function $M: \mathbb{R} \rightarrow \mathcal{M}_{n}(\mathbb{C})$, it will be useful to know how to differentiate it, as well as its matrix powers and invariants. For that purpose, we observed that the following formulas hold (we assume $M$ is an invertible matrix when necessary):

$$
\begin{aligned}
\left(M^{k}\right)^{\prime} & =\sum_{j=0}^{k-1} M^{j} M^{\prime} M^{k-j-1}, k \in \mathbb{N}, \\
(\operatorname{tr}(M))^{\prime} & =\operatorname{tr}\left(M^{\prime}\right), \\
|M|^{\prime} & =\operatorname{tr}\left(\operatorname{adj}(M) M^{\prime}\right)=|M| \operatorname{tr}\left(M^{-1} M^{\prime}\right), \\
\left(M^{-1}\right)^{\prime} & =-M^{-1} M^{\prime} M^{-1} .
\end{aligned}
$$

We also remember that $\operatorname{tr}(A B)=\operatorname{tr}(B A)$ for every $A, B \in \mathcal{M}_{n}(\mathbb{C})$.

\section{Liouville's Formula}

In order to simplify the notation we will define, for convenience:

$$
S_{1}(t):=\sum_{k=0}^{\infty} \frac{E^{k} t^{2 k}}{(2 k) !}, \quad S_{2}(t):=\sum_{k=0}^{\infty} \frac{E^{k} t^{2 k+1}}{(2 k+1) !}
$$

where $E$ is defined as in the statement of Theorem 1 . Observe that $S_{1}$ is even and $S_{2}$ is odd. Then:

$$
\begin{aligned}
X(t)=S_{1}(t)-M_{+} S_{2}(t), & X^{\prime}(t)=E S_{2}(t)-M_{+} S_{1}(t), \\
X(-t) & =S_{1}(t)+M_{+} S_{2}(t), \quad X^{\prime}(-t)=-E S_{2}(t)-M_{+} S_{1}(t),
\end{aligned}
$$

where $M_{+}:=(F+G)^{-1}(A+B)$. In addition, we have:

$$
X^{\prime \prime}=X E, X^{(k+2)}=X^{(k)} E, k \in \mathbb{N} .
$$


Theorem 3 (Abel-Jacobi-Liouville identity). Let $n=2$. Then $\left(|X|,\left|X^{\prime}\right|\right)$ is the unique solution of the system of differential equations:

$$
\begin{aligned}
& x^{\prime \prime}=\operatorname{tr}(E) x+2 y, \\
& y^{\prime \prime}=2|E| x+\operatorname{tr}(E) y,
\end{aligned}
$$

subject to the one point conditions $x(0)=1, y(0)=\left|-M_{+}\right|, x^{\prime}(0)=\operatorname{tr}\left(-M_{+}\right), y^{\prime}(0)=$ $\operatorname{tr}\left(\operatorname{adj}\left(-M_{+}\right) E\right)$.

Proof. First of all, we observe that $|X(0)|=1$ and since $|X|$ is continuous, this means $|X| \neq 0$ in a neighborhood of 0 , so we will assume we work in that neighborhood where $X$ is invertible. Now, we have:

$$
|X|^{\prime}=|X| \operatorname{tr}(Y)
$$

where $Y=X^{-1} X^{\prime}$. From (3) and (4), we have:

$$
Y^{\prime}=E-Y^{2}
$$

Hence, differentiating (6) again and using (7):

$$
|X|^{\prime \prime}=|X|^{\prime} \operatorname{tr}(Y)+|X| \operatorname{tr}\left(E-Y^{2}\right)=|X|^{\prime} \operatorname{tr}(Y)+|X|\left[\operatorname{tr}(E)-\operatorname{tr}\left(Y^{2}\right)\right] .
$$

Now, applying (2) to $Y$, we have:

$$
\operatorname{tr}\left(Y^{2}\right)=\operatorname{tr}(Y)^{2}-2|Y|
$$

Solving for $\operatorname{tr}(Y)$ in (6), taking into account (9) and substituting in (8):

$$
\begin{aligned}
|X|^{\prime \prime} & =\frac{\left(|X|^{\prime}\right)^{2}}{|X|}+|X|\left[\operatorname{tr}(E)-\left(\operatorname{tr}(Y)^{2}-2|Y|\right)\right] \\
& =\frac{\left(|X|^{\prime}\right)^{2}}{|X|}+|X|\left[\operatorname{tr}(E)-\left(\left[\frac{|X|^{\prime}}{|X|}\right]^{2}-2\left|X^{-1}\right|\left|X^{\prime}\right|\right)\right] \\
& =\operatorname{tr}(E)|X|+2\left|X^{\prime}\right| .
\end{aligned}
$$

By (4), we could apply the same process to $\left|X^{\prime}\right|$, and we would get:

$$
\left|X^{\prime}\right|^{\prime \prime}=\operatorname{tr}(E)\left|X^{\prime}\right|+2\left|X^{\prime \prime}\right|=\operatorname{tr}(E)\left|X^{\prime}\right|+2|E||X| .
$$

Thus, it is clear that $\left(|X|,\left|X^{\prime}\right|\right)$ solves the system of equations (5).

Observe $X(0)=\operatorname{Id}$ and $X^{\prime}(0)=-M_{+}$. By (3), we also have $|X|^{\prime}(0)=\operatorname{tr}\left(-M_{+}\right)$and $\left|X^{\prime}\right|^{\prime}(0)=\operatorname{tr}\left(\operatorname{adj}\left(-M_{+}\right) E\right)$, which ends the proof.

Theorem 3 suggests the question of whether this kind of behavior is common to the determinant of the fundamental matrix for any order $n \geq 1$. The following example shows that the system (5) does not hold in general for $n \geq 3$.

Example 1. Let us consider the system:

$$
\begin{aligned}
x^{\prime}(t)+x(-t) & =0, \\
y^{\prime}(t)+y(-t) & =0, \\
z^{\prime}(t)+2 z(-t) & =0,
\end{aligned}
$$

that is, $F=\mathrm{Id}, G=A=0$ and:

$$
B=\left(\begin{array}{lll}
1 & 0 & 0 \\
0 & 1 & 0 \\
0 & 0 & 2
\end{array}\right)
$$


The solution of this system (up to multiplication by constants) is given by $x(t)=y(t)=\cos t-$ $\sin t$ and $z(t)=\cos (2 t)-\sin (2 t)$. Hence:

$$
\begin{aligned}
|X(t)| & =(\cos t-\sin t)^{2}(\cos (2 t)-\sin (2 t)), \\
\left|X^{\prime}(t)\right| & =-2(\sin t+\cos t)^{2}(\sin (2 t)+\cos (2 t)) .
\end{aligned}
$$

On the other hand, the system (5) becomes:

$$
\begin{aligned}
& x^{\prime \prime}=-6 x+2 y \\
& y^{\prime \prime}=-8 x-6 y
\end{aligned}
$$

subject to the one point conditions $x(0)=1, y(0)=-2, x^{\prime}(0)=-4, y^{\prime}(0)=-8$. Observe that the initial conditions are satisfied by $\left(|X|,\left|X^{\prime}\right|\right)$. Nevertheless:

$$
\begin{array}{r}
|X|^{\prime}(t)+6|X|(t)-2\left|X^{\prime}\right|(t)=2 \sin (2 t)+7 \sin (4 t)+6 \cos (2 t)+3 \cos (4 t)+5 \neq 0, \\
\left|X^{\prime}\right|^{\prime}(t)+8|X|(t)+6\left|X^{\prime}\right|(t)=-2(8 \sin (2 t)+\sin (4 t)+4 \cos (2 t)-5 \cos (4 t)+1) \neq 0 .
\end{array}
$$

Hence, $\left(|X|,\left|X^{\prime}\right|\right)$ is not a solution of the system (10).

Regardless of this example, we may wonder whether the following more general statement is true:

For any $n \geq 1$, if $X(t)$ is a fundamental matrix of problem (1), then $|X(t)|$ can be obtained as a component of the solution of a linear system of differential equations with constant coefficients, those coefficients depending only on the different invariants appearing in the Cayley-Hamilton theorem for the matrix $E$, which is defined as in Theorem 1.

Nevertheless, for orders greater than $n=2$, expression (9) does not hold in general, so our calculations will involve a non-trivial product between $X$ and $X^{\prime}$. This difficulty suggests that the statement might not be true.

\section{Cases}

We now illustrate, in some cases, the explicit expression of $|X(t)|$ depending on the values of the parameters. Not all possible cases are present, but the reader can get an idea of the richness of the behavior of $|X(t)|$ :

Case 1: $\operatorname{tr}(E)>0, \operatorname{tr}(E)>2|E|$.

In this case:

$$
\begin{aligned}
|X(t)|= & \frac{1}{|E|}\left[\left(|E|-\left|M_{+}\right|\right) \cosh (\sqrt{\operatorname{tr}(E)} t)\right. \\
& -\frac{|E| \operatorname{tr}\left(M_{+}\right)+\operatorname{tr}\left(\operatorname{adj}\left(M_{+}\right) E\right)}{\sqrt{\operatorname{tr}(E)}} \sinh (\sqrt{\operatorname{tr}(E) t}) \\
& \left.+\left|M_{+}\right| \cosh (\sqrt{\operatorname{tr}(E)-2|E|})-\frac{\operatorname{tr}\left(\operatorname{adj}\left(M_{+}\right) E\right.}{\sqrt{\operatorname{tr}(E)-2|E|}} \sinh (\sqrt{\operatorname{tr}(E)-2|E|} t)\right] .
\end{aligned}
$$

Case 2: $\operatorname{tr}(E)=0,|E|<0$ :

$$
\begin{aligned}
|X(t)|= & \frac{2\left|M_{+}\right| \sqrt{-|E|} \cosh (\sqrt{-2|E|} t)+\sqrt{2} \operatorname{tr}\left(\operatorname{adj}\left(M_{+}\right) E\right) \sinh (\sqrt{-2|E|} t)}{2|E| \sqrt{-2|E|}} \\
& +\frac{\left|M_{+}\right|+\operatorname{tr}\left(\operatorname{adj}\left(M_{+}\right) E\right) t}{|E|}-\operatorname{tr}\left(M_{+}\right) t+1
\end{aligned}
$$

Case 3: $\operatorname{tr}(E)=0,|E|>0$ : 


$$
\begin{aligned}
|X(t)|= & \frac{\operatorname{tr}\left(\operatorname{adj}\left(M_{+}\right) E\right) \sin (\sqrt{2} \sqrt{|E|} t)}{\sqrt{2}|E|^{3 / 2}} \\
+ & \frac{-\left|M_{+}\right|-\operatorname{tr}\left(M_{+}\right)|E| t+|E|-\operatorname{tr}\left(\operatorname{adj}\left(M_{+}\right) E\right) t}{|E|} \\
& +\frac{\left|M_{+}\right| \cos (\sqrt{2} \sqrt{|E|} t)}{|E|} .
\end{aligned}
$$

Case 4: $|E|=0, \operatorname{tr}(E)>0$ :

$$
\begin{aligned}
|X(t)|= & \frac{\left[\operatorname{tr}(E)\left(\beta-\left|M_{+}\right| t\right)+\operatorname{tr}\left(\operatorname{adj}\left(M_{+}\right) E\right)\right] \sinh (\sqrt{\operatorname{tr}(E)} t)}{\operatorname{tr}(E)^{3 / 2}} \\
& +\frac{\left[\operatorname{tr}(E)-\operatorname{tr}\left(\operatorname{adj}\left(M_{+}\right) E\right) t\right] \cosh (\sqrt{\operatorname{tr}(E)} t)}{\operatorname{tr}(E)} .
\end{aligned}
$$

Case 5: $|E|=0, \operatorname{tr}(E)=0$ :

$$
|X(t)|=-\frac{\operatorname{tr}\left(\operatorname{adj}\left(M_{+}\right) E\right)}{3} t^{3}-\left|M_{+}\right| t^{2}-\operatorname{tr}\left(M_{+}\right) t+1 .
$$

This is the only case for which we can explicitly compute the zeros of $|X|(t)$, having at least one real root assuming $\operatorname{tr}\left(\operatorname{adj}\left(M_{+}\right) E\right) \neq 0$.

\section{Application: Square Roots of the Harmonic Oscillator}

In [10], the authors used the reflection operator to compute a square root of the classical harmonic oscillation equation. The same can be done for a pair of coupled harmonic oscillators (see, for instance, ref. [17]). In this case, we are talking about a linear system of order two of the form:

$$
x^{\prime \prime}(t)+M x(t)=0,
$$

where $M$ is a symmetric matrix with positive eigenvalues. Such a matrix admits a real square root (see [12]) that is, a real matrix $P$ such that $P^{2}=M$. If $\lambda_{1}>\lambda_{2}>0$ are the eigenvalues of $M$ and:

$$
M=\left(\begin{array}{ll}
a & c \\
c & b
\end{array}\right)
$$

we can take $P$ such that $\left(\sqrt{(a-b)^{2}+4 c^{2}}\right) P$ equals

$$
\left(\begin{array}{cc}
\frac{1}{2}\left(\sqrt{\lambda_{2}}\left(\lambda_{1}-2 a\right)+\left(\lambda_{1}-2 b\right) \sqrt{\lambda_{1}}\right) & c\left(\sqrt{\lambda_{1}}-\sqrt{\lambda_{2}}\right) \\
c\left(\sqrt{\lambda_{1}}-\sqrt{\lambda_{2}}\right) & \frac{1}{2}\left(\sqrt{\lambda_{1}}\left(\lambda_{1}-2 a\right)+\left(\lambda_{1}-2 b\right) \sqrt{\lambda_{2}}\right)
\end{array}\right)
$$

Consider now the operator $L=D+P \varphi^{*}$, where $D$ is the derivative and $\varphi^{*}$ is the pullback by the reflection, that is, $\varphi^{*}(f)(t)=f(-t)$ (cf. [12]). Then we have that:

$$
L^{2}=\left(D+P \varphi^{*}\right)^{2}=D^{2}+P^{2},
$$

so $L$ is a square root of (11).

We can consider the equation:

$$
L x(t)=x^{\prime}(t)+P x(-t)=0,
$$


which provides a factorization of the harmonic oscillator different from the Dirac equation [18] (see [12]). By Theorem 1, the fundamental matrix of (13) is given by

$$
X(t)=\cos P t-\sin P t .
$$

Moreover, the determinant of this matrix is given by Theorem 3 as the first component of the solution of the system of differential equations:

$$
\begin{aligned}
& x^{\prime \prime}=-\left(\lambda_{1}+\lambda_{2}\right) x-2 y, \\
& y^{\prime \prime}=-2 \lambda_{1} \lambda_{2} x-\left(\lambda_{1}+\lambda_{2}\right) y,
\end{aligned}
$$

subject to the one point conditions:

$$
\begin{array}{ll}
x(0)=1, & x^{\prime}(0)=-\left(\sqrt{\lambda_{1}}+\sqrt{\lambda_{2}}\right), \\
y(0)=\sqrt{\lambda_{1} \lambda_{2}}, & y^{\prime}(0)=\lambda_{2} \sqrt{\lambda_{1}}+\lambda_{1} \sqrt{\lambda_{2}} .
\end{array}
$$

That is:

$$
|X(t)|=-\frac{\left(\sqrt{\lambda_{1}}+\sqrt{\lambda_{2}}\right) \sin \left(\left[\sqrt{\lambda_{1}}-\sqrt{\lambda_{2}}\right] t\right)}{\sqrt{\lambda_{1}}-\sqrt{\lambda_{2}}}+\cos \left(\left[\sqrt{\lambda_{1}}+\sqrt{\lambda_{2}}\right] t\right) .
$$

Author Contributions: These authors contributed equally to this work. All authors have read and agreed to the published version of the manuscript.

Funding: F. Adrián F. Tojo was partially supported by Xunta de Galicia, project ED431C 2019/02, and by the Agencia Estatal de Investigación (AEI) of Spain under grant MTM2016-75140-P, cofinanced by the European Community fund FEDER.

Institutional Review Board Statement: Not applicable.

Informed Consent Statement: Not applicable.

Data Availability Statement: Not applicable.

Conflicts of Interest: The authors declare no conflict of interest.

\section{References}

1. Cabada, A.; Infante, G.; Tojo, F.A.F. Nontrivial solutions of Hammerstein integral equations with reflections. Bound. Value Probl. 2013, 2013, 1-22. [CrossRef]

2. Gupta, C.P. Existence and uniqueness theorems for boundary value problems involving reflection of the argument. Nonlinear Anal. 1987, 11, 1075-1083. [CrossRef]

3. O'Regan, D. Existence results for differential equations with reflection of the argument. J. Aust. Math. Soc. 1994, 57, 237-260. [CrossRef]

4. Aftabizadeh, A.R.; Huang, Y.K.; Wiener, J. Bounded solutions for differential equations with reflection of the argument. J. Math. Anal. Appl. 1988, 135, 31-37. [CrossRef]

5. Cabada, A.; Fernández Tojo, F.A. Periodic solutions for some phi-Laplacian and reflection equations. Bound. Value Probl. 2016, 56, 2016. [CrossRef]

6. Sadybekov, M.; Sarsenbi, A. Criterion for the basis property of the eigenfunction system of a multiple differentiation operator with an involution. Differ. Equ. 2012, 48, 1112-1118. [CrossRef]

7. Kopzhassarova, A.; Sarsenbi, A. Basis properties of eigenfunctions of second-order differential operators with involution. Abstr. Appl. Anal. 2012, 2012, 576843. [CrossRef]

8. Cabada, A.; Tojo, F.A.F. Existence results for a linear equation with reflection, non-constant coefficient and periodic boundary conditions. J. Math. Anal. Appl. 2014, 412, 529-546. [CrossRef]

9. Cabada, A.; Tojo, F.A.F. Solutions and Green's function of the first order linear equation with reflection and initial conditions. Bound. Value Probl. 2014, 2014, 99. [CrossRef]

10. Cabada, A.; Tojo, F.A.F. Comparison results for first order linear operators with reflection and periodic boundary value conditions. Nonlinear Anal. 2013, 78, 32-46. [CrossRef]

11. Cabada, A.; Tojo, F.A.F. Solutions of the first order linear equation with reflection and general linear conditions. Glob. J. Math. Sci. 2013, 2, 1-8. 
12. Cabada, A.; Tojo, F.A.F. Green's functions for reducible functional differential equations. Bull. Malays. Math. Sci. Soc. 2017, 40, 1071-1092. [CrossRef]

13. Cabada, A.; Tojo, F.A.F. On linear differential equations and systems with reflection. Appl. Math. Comput. 2017, 305, 84-102. [CrossRef]

14. Codesido, S.; Tojo, F.A.F. Differential systems with reflection and matrix invariants. Math. Methods Appl. Sci. 2018, 41, 7399-7406. [CrossRef]

15. Tojo, F.A.F. Green's functions of recurrence relations with reflection. J. Math. Anal. Appl. 2019, 477, 1463-1485. [CrossRef]

16. Tojo, F.A.F.; Torres, P.J. Green's Functions of Partial Differential Equations with Involutions. J. Appl. Anal. Comput. 2017, 7, 1127-1138.

17. Ren, W. Synchronization of coupled harmonic oscillators with local interaction. Automatica 2008, 44, 3195-3200. [CrossRef]

18. Dirac, P.A.M. The quantum theory of the electron. Proc. R. Soc. Lond. A 1928, 117, 610-624. 